${ }^{1}$ Klinik und Poliklinik für Angiologie, Departement Herz und Gefäße, Inselspital, Universität Bern, Schweiz.

\section{Schlüsselwörter:}

Varikose - Thrombophlebitis - Venenthrombose $\cdot$ Chronische venöse Insuffizienz Postthrom botisches Syndrom . Duplexsonographie. Doppler · Phlethysmographie $\cdot$ Phlebographie

Herz 2007;32:10-7

s00059-007-2958-3

\section{Key Words:}

Varicose reins - Thrombophlebitis - Deep vein thrombosis . Venous insufficiency. syndrome - Ultrasonography - Doppler . Plethysmography . Phlebography

\title{
Diagnostik venöser Erkrankungen
}

\author{
Dai-Do Do, Marc Husmann ${ }^{1}$
}

DOI 10.1007/

\section{Zusammenfassung}

Die venösen Erkrankungen der unteren Extremitäten stellen mit der Varikose, der Thrombophlebitis, der tiefen Beinvenenthrombose und der chronischen venösen Insuffizienz aufgrund des weitverbreiteten und im Alter zunehmenden Vorkommens relevante Krankheitsbilder hinsichtlich Diagnostik und Therapie dar. Die Diagnose der Varikose und Thrombophlebitis kann in der Mehrheit der Fälle klinisch gestellt werden, und apparative Diagnostik, meist Ultraschall, wird zur Dokumentation, für das Therapiekonzept oder zur Operationsplanung benötigt. Umgekehrt kann die tiefe Beinvenenthrombose klinisch nicht sicher diagnostiziert werden und bedarf einer der klinischen Wahrscheinlichkeit entsprechenden adäquaten Diagnostik. Die farbkodierte Duplexsonographie und die Kompressionssonographie sind in geübten Händen als nichtinvasive Verfahren zu den Methoden der Wahl avanciert. Goldstandard bleibt jedoch wegen der geringsten Untersucherabhängigkeit die Phlebographie, welche in der klinischen Routine weitgehend durch nichtinvasive

\section{Diagnosis of Venous Disease} Postthrombotic

\begin{abstract}
Venous disease of the lower extremities comprises several common conditions such as varicose veins, superficial thrombophlebitis, deep venous thrombosis and chronic venous insufficiency. The high prevalence and increasing incidence with age of these disorders impose relevant diagnostic and therapeutic concerns. Varicose veins and superficial thrombophlebitis are easily diagnosed clinically and additional diagnostic means, i.e., duplex scan, is needed for documentation or planning of surgery. Deep venous thrombosis cannot be confirmed clinically and further imaging based on clinical probability is usually required for correct diagnosis. Color-coded duplex and compression sonography have emerged as the means of choice in skilled hands. Phlebography still remains the gold standard based on its investiga-
\end{abstract}

Verfahren abgelöst wurde. Die Spiral-Computertomographie wie auch die Kernspintomographie sind wegen der Kosten nicht für die breite Anwendung geeignet, können aber bei diagnostischer Unsicherheit, vor allem bei Beckenvenenthrombosen, sinnvoll eingesetzt werden. Im Vergleich zur Phlebographie ergeben die Schichtbilder wie auch die Duplexsonographie zusätzliche Informationen über die die Gefäße umgebenden Strukturen und können allenfalls eine Differentialdiagnose sichern. Die Computertomographie und die Phlebographie sind durch ihre Kontrastmittelund Strahlungsbelastung limitiert. Die chronische venöse Insuffizienz kann durch eine genaue Anamneseerhebung und klinische Untersuchung meist relativ sicher festgestellt werden. Funktionelle und morphologische Untersuchungsmethoden wie die Plethysmographie, Duplexsonographie und Phlebographie eignen sich hier je nach klinischer Fragestellung zur Sicherung der Diagnose, zur Evaluation einer operativen Sanierung der Venen und zur Bestimmung der Ätiologie der chronischen venösen Insuffizienz.

tor-independent characteristics. However, it is becoming more and more a research tool. CT scan and magnetic resonance imaging are too expensive for broad application, but may be helpful in cases of uncertainty such as iliac vein thrombosis. CT scans, magnetic resonance imaging and sonography reveal additional information compared to phlebography about surrounding tissue and may help in establishing a differential diagnosis. Limitations of CT scan and phlebography are radiation exposure and contrast media application. Chronic venous insufficiency is basically established by medical history and clinical findings. Functional and imaging tests such as plethysmography, color-coded duplex sonography and phlebography are essential for confirming the diagnosis, evaluating a surgical intervention or defining the cause of chronic venous insufficiency. 


\section{Einleitung}

Die venösen Erkrankungen der unteren Extremitäten nehmen aufgrund ihrer Prävalenz und des zunehmenden Alters der Bevölkerung einen beachtlichen Platz im ambulanten und stationären Krankengut ein. Obwohl Anamnese und klinische Untersuchung in einem diagnostischen Stufenplan dabei nach wie vor an erster Stelle stehen, ist heutzutage anerkannt, dass dies zur Festlegung der adäquaten Therapie nicht immer genügt. In der Diagnostik von Krankheiten sowohl des oberflächlichen als auch des tiefen Venensystems hat sich inzwischen vor allem die Duplexsonographie anstelle der invasiven phlebographischen Methode durchgesetzt. Die letztgenannte Untersuchung sowie andere invasive Untersuchungstechniken wie beispielsweise die blutige Venendruckmessung haben noch wissenschaftliche Bedeutung, vor allem in der Erforschung neuer chirurgischer rekonstruktiver Eingriffe.

\section{Varikose}

Je nach geographischer Herkunft und Definition beträgt die Prävalenz dieser als dilatiert, geschlängelt und elongiert veränderten subkutanen Venen zwischen $10 \%$ und $50 \%$ [1,2]. Bei Individuen > 60 Jahre ist eine Varikose fast immer zu finden. Im Gegensatz zur primären Varikose können ätiologisch bei sekundärer Varikose Ursachen ermittelt werden: am häufigsten wegen Verlegung der tiefen Leitvenensystems nach tiefer Venenthrombose (TVT), seltener Angiodysplasie oder arteriovenöse Fisteln. Anhand der Erscheinungsform werden unterschieden:

- Stammvarize: betrifft den Hauptstamm und/oder die großen Nebenäste in bis zu $80 \%$ der Fälle der V. saphena magna und in $20 \%$ der Fälle der V. saphena parva mit einem Durchmesser $>4$ mm (Abbildung 1);

- retikuläre Varize: dilatierte, nicht palpable subdermale Venen mit einem Durchmesser $<4$ mm;

- Teleangiektasie: dilatierte intradermale Venulen mit einem Durchmesser $<1 \mathrm{~mm}$.

\section{Diagnostik}

Viele Patienten mit Varikose bleiben asymptomatisch. Aufgrund der Basler Studie ist bekannt, dass $55 \%$ der Patienten mit Varizen über Beinbeschwerden klagen, wie sie auch von $45 \%$ Venengesunder geäußert werden [3]. Neben Schwellungsneigung vor allem nach längerem Stehen werden Schweregefühl, Spannungsgefühl, Müdigkeit und Druckgefühl in den Unterschenkeln angegeben. Während Juckreiz gelegentlich auftritt, sind Wadenkrämpfe nicht unbedingt ein typisches Symptom.

Die klinische Beurteilung sollte zuerst beim stehenden, dann liegenden Patienten erfolgen und be- inhaltet die Inspektion sowie Palpation der $\mathrm{Vv}$. saphenae. Es wird geprüft, ob Ödeme, trophische Läsionen und Hautveränderungen vorliegen. Mit der Verfügbarkeit der Continuous-Wave-(CW-)Doppler bzw. der Duplexsonographie haben die klinisch-phlebologischen Tests wie z.B. der Trendelenburg-Test an Bedeutung verloren. Die Untersuchung mit einem einfachen Taschen-CW-Doppler liefert einen sicheren Nachweis einer Klappeninsuffizienz im Verlauf der Stammvenen, verpasst allerdings im Vergleich zur Duplexsonographie in bis zu 30\% der Fälle wichtige Informationen vor allem über die Mündungsstelle der Varizen in die tiefen Venen [4]. Neben der genauen Morphologie liefert die Duplexsonographie auch funktionelle Informationen zur Varikose. Venöser Reflux durch insuffiziente Klappen kann unter standardisierten Untersuchungsbedingungen ermittelt werden [5]. Eine Refluxzeit $>0,5 \mathrm{~s}$ ist als pathologisch anzusehen [6, 7]. Die Phlebographie bzw. Varikographie ist bei der Varikosediagnostik kaum noch indiziert und wird gelegentlich bei Rezidivvarikose vor Reoperation durchgeführt.

\section{Thrombophlebitis}

Die Varikophlebitis macht bis zu $90 \%$ der oberflächlichen Thrombophlebitiden aus. Die Diagnose lässt sich meist klinisch und einfach stellen (Abbil-

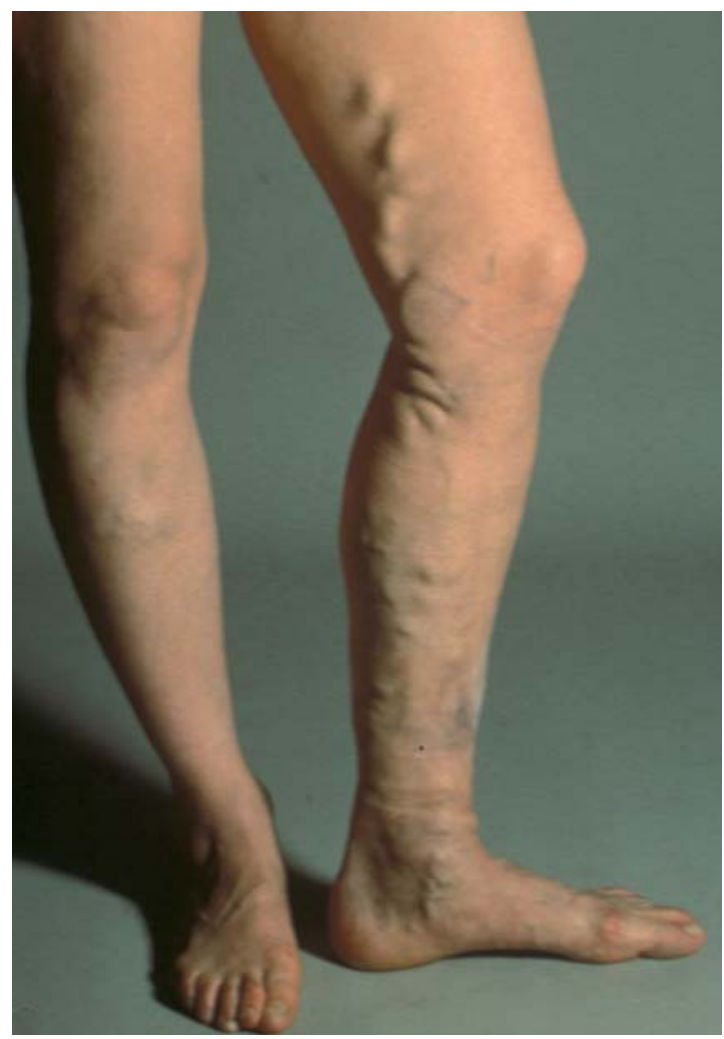

Abbildung 1. Stammvarize der dilatierten, geschlängelten und elongierten $\mathrm{V}$. saphena magna links.

Figure 1. Varicosity of the long saphenous vein (left limb). 


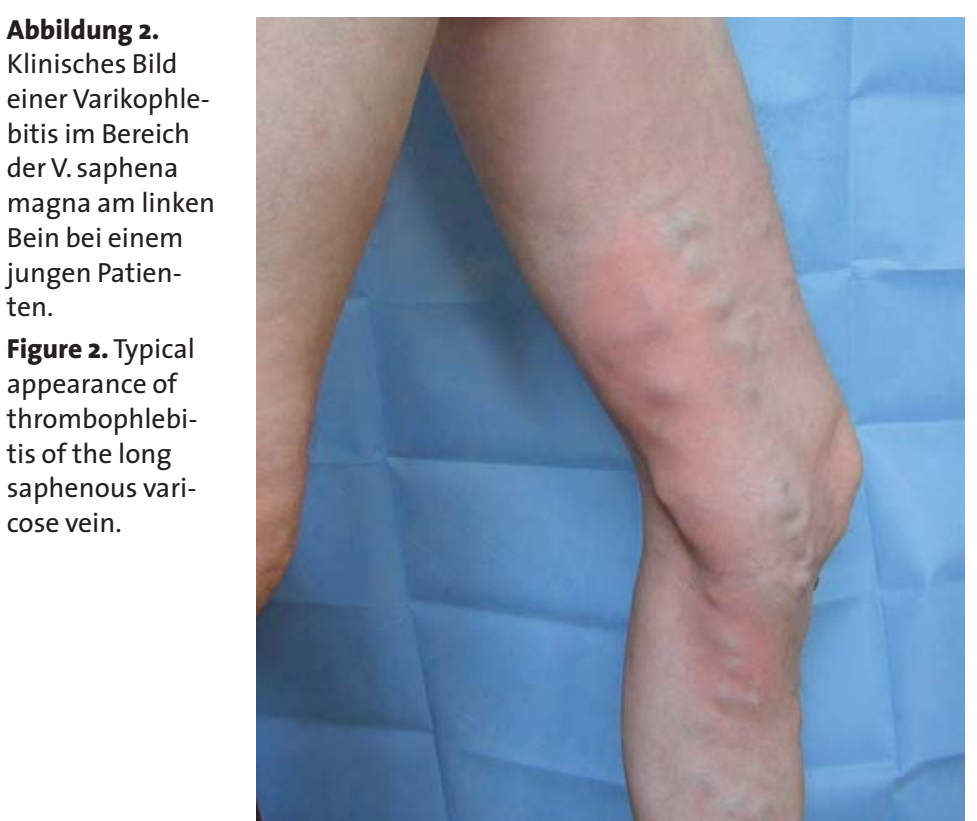

dung 2). Das embolische Risiko ist klein, es sei denn, es liegt eine Ausdehnung Richtung Mündungsstelle und somit in das tiefe Venensystem vor [8]. Deshalb empfiehlt sich zur weiteren Abklärung eine Duplexsonographie, um die genaue Ausdehnung beurteilen und allfällige begleitende tiefe Phlebothrombosen ausschließen zu können. Im Fall von Throm-
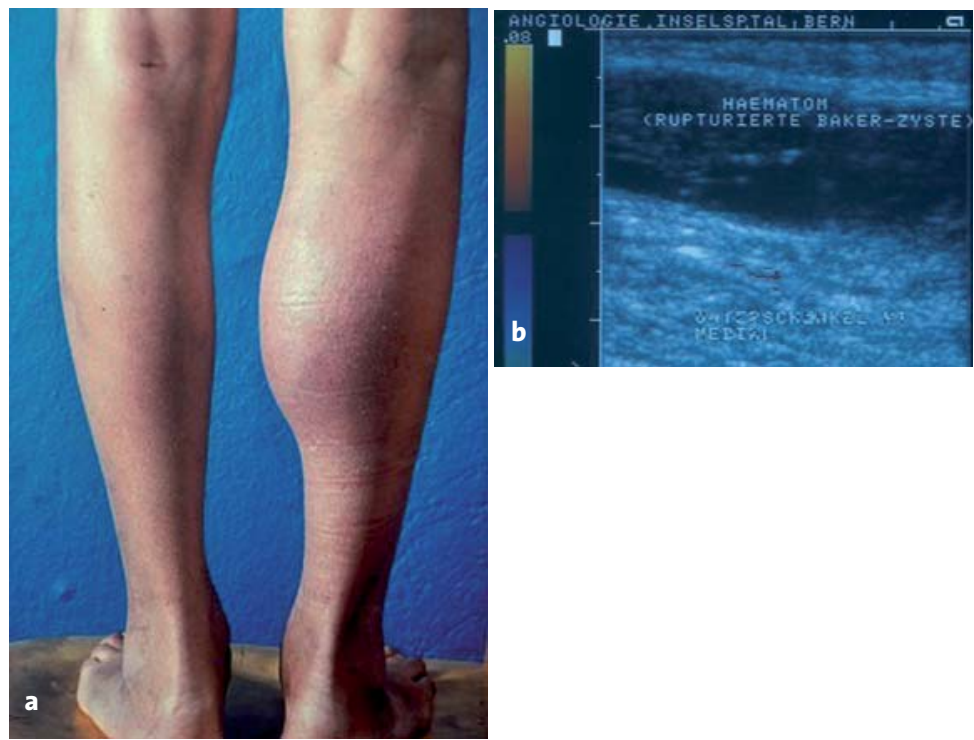

Abbildungen 3a und $\mathbf{3 b}$. a) Rupturierte Baker-Zyste bei einem Patienten mit Verdachtsdiagnose einer tiefen Venenthrombose am rechten Bein als Einweisungsgrund. b) Ausgedehntes Hämatom am rechten Unterschenkel infolge der rupturierten Baker-Zyste.

Figures $\mathbf{3}$ a and $\mathbf{3}$ b. a) Ruptured Baker cyst in a young patient with primarily suspected deep vein thrombosis. b) Ultrasonographic imaging of hematoma in the right calf as consequence of the ruptured Baker cyst. bophlebitiden in augenscheinlich gesunden Venen sollte diagnostisch nach Thrombophilie oder dem Vorliegen eines Malignoms gesucht werden [9-12].

\section{Tiefe Venenthrombose}

Die jährliche Inzidenz der TVT der unteren Extremität bei der allgemeinen Bevölkerung in den entwickelten Ländern beträgt 1-2 auf 1000 [13].

Als Folge treten im akuten und subakuten Stadium häufig Lungenembolien auf, und zwar in $>50 \%$ der Fälle bei Vorliegen einer proximalen Phlebothrombose und in $>25 \%$ der Fälle bei Vorliegen einer distalen Beinvenenthrombose [14]. Mittel- und langfristig ist zudem als Folge des postthrombotischen Syndroms mit einer Inzidenz um 15\% nach 5 Jahren, 20\% nach 10 Jahren und 27\% nach 20 Jahren eine chronische venöse Insuffizienz zu erwarten [15]. Um fatale Lungenembolien $\mathrm{zu}$ vermeiden bzw. den Schweregrad der Spätfolgen zu verringern, ist eine akkurate Diagnose der TVT im Hinblick auf eine baldige Therapieeinleitung gefragt. Des Weiteren ist daran zu erinnern, dass wegen der abnehmenden Prävalenz in der Patientenpopulation mit Verdacht auf eine TVT nur in $<25 \%$ der Fälle auch tatsächlich eine solche vorliegt [16]. $\mathrm{Zu}$ hohe falsch positive Diagnosen würden die Rate der Blutungskomplikationen bei der Behandlung erhöhen.

\section{Klinische Diagnostik}

Die klinische Diagnose ist unzuverlässig. Schmerzen und Schwellung der unteren Extremitäten können verschiedene Ursachen haben (Abbildungen 3a und 3b). In einer Studie mit 160 konsekutiven Patienten mit Verdacht auf Phlebothrombose fanden Hull et al. bei negativem Phlebogramm folgende Ursachen [17]:

- Muskelzerrung, Muskelriss, Torsionstrauma des Beins:

$40 \%$,

- Schwellung in einem paralysierten Bein: $\quad 9 \%$,

- Lymphödem oder Lymphangitis: 7\%,

- chronische venöse Insuffizienz: 7\%,

- Baker-Zyste:

$5 \%$,

- Zellulitis:

$3 \%$,

- Knieabnormität: $2 \%$,

- unbekannte: $26 \%$.

Dazu können noch andere lokale Ursachen wie Fraktur, Hämatom, Kompartmentsyndrom oder arterielle Ischämie sowie systemische Ursachen wie Herzinsuffizienz oder Hypoproteinämie usw. gerechnet werden. Um diese große Anzahl an Patienten mit Verdacht auf, aber in der Tat ohne TVT von unnötigen, meist kostspieligen, spezifischen diagnostischen Untersuchungen auszuschließen, wur- 
den klinische Modelle zur Abschätzung entwickelt, ob eine geringe, mittlere oder hohe Wahrscheinlichkeit für das Vorliegen einer TVT besteht [18]. Eines dieser Wahrscheinlichkeitsmodelle, der Wells-Score (Tabelle 1), wird am häufigsten benutzt [19].

\section{Apparative Diagnostik}

Labormäßig weisen die Plasma-D-Dimere, spezifische Abbauprodukte von Fibrin, indirekt auf Thromboembolie hin. Eine hohe D-Dimer-Konzentration ist allerdings auch bei anderen Erkrankungen anzutreffen, so dass sich der Test wegen der niedrigen Spezifität um $40 \%$ nicht zum positiven Nachweis von TVTs eignet. Bei einem D-Dimer-Spiegel $<500 \mathrm{ng} / \mathrm{ml}$ beträgt hingegen der negative prädiktive Wert $>98 \%$, was zusammen mit der Klinik den Ausschluss der TVT-Diagnose erlaubt [20-22].

\section{Bildgebende Verfahren}

Zum positiven Nachweis einer TVT eignen sich ausschließlich bildgebende Verfahren:

- Ultrasonographie: B-Bild-Kompressions-/Duplexsonographie,

- Phlebographie,

- Spiral-Computertomographie (CT),

- Kernspintomographie (MRT).

Die Spiral-CT und in zweiter Linie die MRT werden dann eingesetzt, wenn im Becken- und Abdominalbereich mit den beiden ersten Verfahren keine eindeutige Diagnose gestellt werden kann und/oder wenn eine Raumforderung als Ursache für eine Venenkompression oder als Begleiterkrankung in Frage kommt [23, 24]. Aus Kostengründen ist allerdings ein breiter Einsatz dieser zwei Verfahren sicher nicht angezeigt.

Phlebographie. Die Phlebographie wird heutzutage nur dann eingesetzt, wenn nichtinvasive Untersuchungsmethoden nicht verfügbar oder deren Resultate zweifelhaft sind.

Der Informationsgehalt eines Phlebogramms wird in seinem Umfang bis heute durch kein anderes Untersuchungsdokument erreicht. Die Methode erlaubt eine eher untersucherunabhängige Befundung und bedarf eines relativ geringen Zeitaufwands. Die Nachteile wie Invasivität, Strahlenexposition, mögliche Kontrastmittelunverträglichkeit oder kontrastmittelinduzierte Niereninsuffizienz bleiben trotz moderner Entwicklung der digitalen Technik und nichtionischer Kontrastmittel bestehen. Viele Autoren gehen nach wie vor davon aus, dass die ,lege artis" durchgeführte Phlebographie und Interpretation der Phlebogramme auch heute noch als Referenzmethode gelten, an der die anderen Methoden ge-

Tabelle 1. Klinisches Wahrscheinlichkeitsmodell nach Wells. TVT: tiefe Venenthrombose.

Table 1. Pretest probability scoring system (Wells score) for deep vein thrombosis (TVT).

Klinisches Symptom Score

Aktives Malignom (unter Therapie oder innerhalb der letzten 6 Monate oder palliative Therapie)

1

Paralyse, Parese oder kürzliche Immobilisation

Kürzliche Bettlägerigkeit $>3$ Tage oder größerer chirurgischer Eingriff in den letzten 4 Wochen

Lokalisierte Druckempfindlichkeit entlang dem tiefen Venensystem

Schwellung des ganzen Beins

Unterschied im Wadenumfang von $>3 \mathrm{~cm}$ zwischen beiden Beinen (gemessen $10 \mathrm{~cm}$ unter der Tuberositas tibiae)

Eindellbares Ödem

Kollateralvenen vorhanden (keine Varikose)

\begin{tabular}{ll}
\hline Interpretation & \\
\hline Hohe Wahrscheinlichkeit einer TVT (75-85\%) & $\geq 3$ \\
Mittlere Wahrscheinlichkeit einer TVT (17-33\%) & $1-2$ \\
Geringe Wahrscheinlichkeit einer TVT (3-10\%) & $(-2)-0$ \\
\hline
\end{tabular}

messen werden [25]. Es darf aber nicht vergessen werden, dass auch die Phlebographie u.a. wegen prinzipieller, sich aus der Anatomie des Venensys-
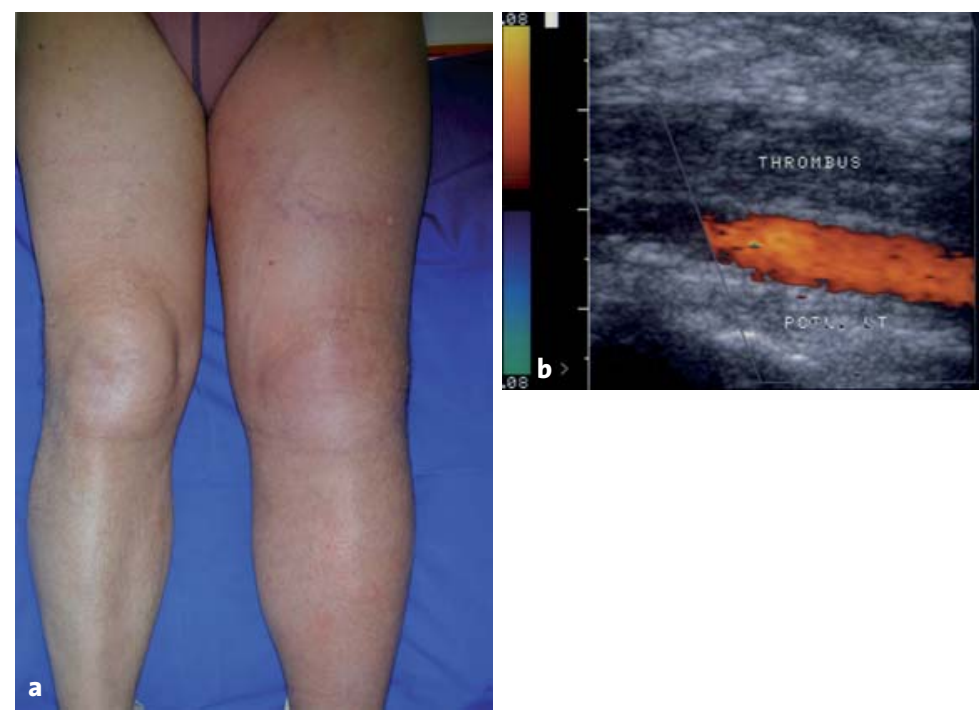

Abbildungen 4a und $\mathbf{4 b}$. a) Junge Patientin mit einer tiefen Venenthrombose am linken Bein. b) Duplexsonographie: vollständige Thrombose bis in die Beckenvenen, hier im Längsschnitt dargestellt die thrombosierte V. poplitea direkt neben der Arterie.

Figures $\mathbf{4 a}$ and $\mathbf{4} \mathbf{b}$. a) Young female patient with deep vein thrombosis of the left limb. b) Duplex scan confirming the diagnosis with the presence of an echogenic thrombus in the popliteal vein (in longitudinal section) beside the patent popliteal artery (in red). 


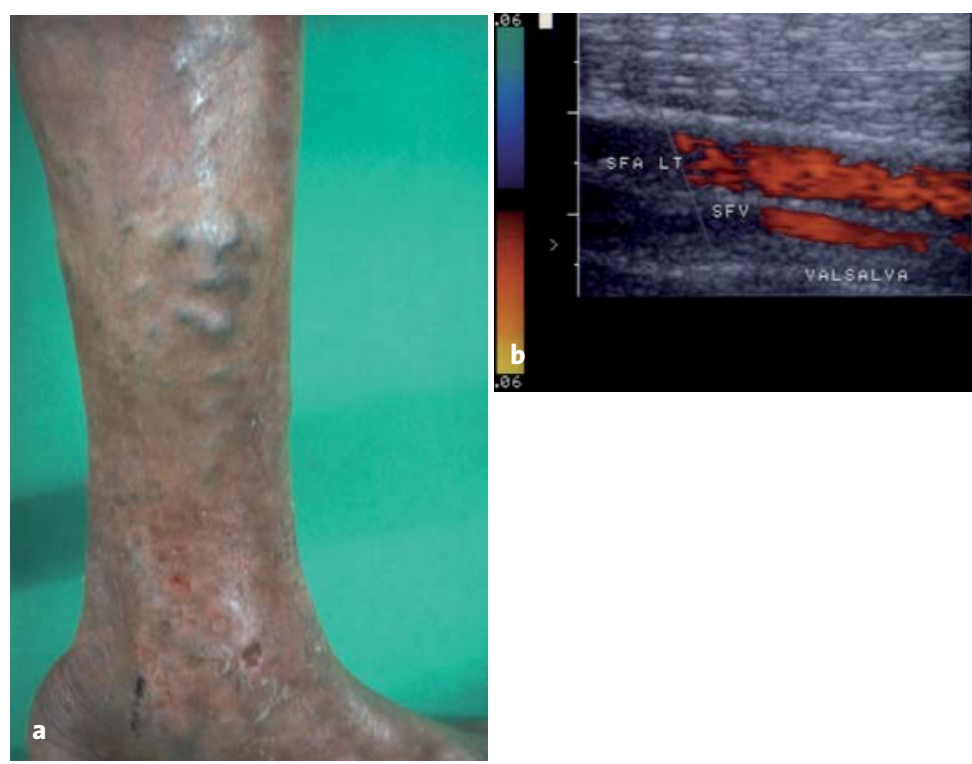

Abbildungen 5a und 5b. a) Chronische venöse Insuffizienz mit sekundärer Varikose und Ulzera am linken Bein bei einer Patientin mit Zustand nach tiefer Venenthrombose vor Jahren. b) Duplexsonographie: postthrombotische Veränderungen in der rekanalisierten, aber lumenreduzierten V. femoralis superior (SFV) mit eindeutigem Reflux bei Valsalva-Pressversuch.

Figures $\mathbf{5 a}$ and $\mathbf{5 b}$. a) Postthrombotic syndrome with secondary varicosis and ulcers of the left leg of a female patient with deep vein thrombosis years ago. $b$ ) Duplex scan demonstrating postthrombotic changes of the left superficial femoral vein (SFV): recanalized, but narrow vessel lumen with pathologic reflux during Valsalva maneuver.

Tabelle 2. Stadieneinteilung nach Widmer.

Table 2. Widmer clinical classification of chronic venous insufficiency.

Stadium I Corona phlebectatica, Ödem, dilatierte epifasziale Venen

Stadium II Hyper- oder Depigmentation, Ekzem, Dermatosklerose

Stadium III Ulkus, florid oder abgeheilt
TVT eine Sensitivität um $95 \%$ und Spezifität um 97\%, für die Unterschenkel-TVT um 90\% bzw. 92\% [27-31]. Die Ultrasonographie ist somit eine wertvolle diagnostische Untersuchungsmethode für TVTs, vor allem wenn sie mit den Resultaten des D-Dimer-Tests und des klinischen Wahrscheinlichkeitsmodells kombiniert wird [22].

B-Bild- oder Duplexsonographie und Phlebographie haben bezüglich des Nachweises einer klinisch relevanten Thrombose den gleichen Stellenwert. Falls die Möglichkeit einer B-Bild-Kompressions- oder Duplexsonographie besteht, sollte sie als Methode der ersten Wahl durchgeführt werden. Diese wegen ihrer einfachen technischen Voraussetzungen leicht zugängigen, relativ kostengünstigen Ultraschallmethoden sind für die Patienten nicht belastend, zeigen im Gegensatz zur Phlebographie auch die perivaskulären Strukturen und erlauben daher eine vielseitige differentialdiagnostische Abgrenzung. Als Nachteile müssen die starke Untersucherabhängigkeit und die nur inkomplette Dokumentationsmöglichkeit gelten. Bei schlechten Bedingungen wie ausgeprägter Adipositas, Ödemen, dem Vorliegen eines Gipsverbands oder größerer Wunden etc. ist die Untersuchung nur bedingt oder gar nicht möglich.

\section{Chonische venöse Insuffizienz}

Bei der chronischen venösen Insuffizienz der unteren Extremitäten handelt es sich um Symptome oder Gewebeveränderungen, die durch eine venöse Hypertonie infolge morphologischer oder funktioneller Abnormitäten der Venen bedingt sind. Pathophysiologisch spielt bei der Entstehung der venösen Hypertonie die primäre Klappeninsuffizienz in $70-80 \%$ die Hauptrolle, in $18-25 \%$ der Fälle entsteht die Klappeninsuffizienz sekundär nach einem Trauma oder einer TVT [32, 33]. Als weitere Ursachen sind venöse Abflussbehinderung oder fehlende Pumpfunktion der Wadenmuskulatur zu erwähnen. In der westlichen Welt ist die chronische venöse Insuffizienz mit einer Prävalenz von bis zu 15\% der Bevölkerung aufgrund der Kosten für diagnostische und therapeutische Maßnahmen sowie wegen Arbeitsausfall von großer sozioökonomischer Bedeutung [34, 35]. Die Inzidenz der chronischen venösen Insuffizienz und deren schwerster Folge, des Ulcus cruris venosum, nimmt mit zunehmendem Alter, Übergewicht, Zustand nach Unfall der betreffenden Extremität und vor allem durchgemachten Thrombophlebitiden oder TVT zu [36].

\section{Klinische Diagnostik}

Die klinische Diagnose der chronischen venösen Insuffizienz ist aufgrund von Beinödemen, Haut- 
veränderungen und Ulzera an den typischen Stellen in den meisten Fällen gut möglich (Abbildung 5a). Während sich die Klassifikation der chronischen venösen Insuffizienz nach Widmer (Tabelle 2) ausschließlich auf die Befunde an der Haut stützt [37], werden bei der neuen CEAP-Klassifikation (Tabelle 3) sowohl die klinischen Befunde als auch die Ätiologie, die anatomischen Veränderungen und die Pathophysiologie berücksichtigt [37-39].

Die genaue Anamneseerhebung sowie die klinische Untersuchung sind von großer Bedeutung, weil sich dadurch die Weichen betreffend Diagnose und damit adäquate Therapie einer chronischen venösen Insuffizienz als häufigste Ursache des Ulcus cruris stellen lassen (Tabelle 4). Die häufigsten Differentialdiagnosen können gezielt ausgeschlossen werden [40].

Wie in der Diagnostik der Varikose haben die klinisch-phlebologischen Tests wie z.B. der Trendelenburg-Test mit der Verfügbarkeit der CW-Doppler, vor allem der Duplexsonographie, nur noch historischen Wert, da sie nicht einfach durchführbar sowie ihre Zuverlässigkeit, Genauigkeit und Sensitivität unbekannt sind.

Apparative Diagnostik - funktionelle Tests Plethysmographien. Diese nichtinvasiven Tests erfassen Veränderungen des Blutvolumens und wurden u.a. zur Beurteilung des Schweregrades der chronischen venösen Insuffizienz entwickelt. Bei der Photoplethysmographie wird das Ausmaß der reflektierenden Lichtanteile ermittelt, welche vom Blutgehalt des Gewebes abhängig sind. Die Methode wird zur Beurteilung der globalen Funktion der venösen Muskelpumpe eingesetzt und ist wertvoll bei der Bestimmung, ob eine chronische venöse Insuffizienz vorliegt. Sie ist allerdings nicht kalibrierbar, und die Resultate sind nur eingeschränkt reproduzierbar. Durch Bestimmung der ve-
Tabelle 3. CEAP-Klassifikation (modifiziert nach [38, 39]).

Table 3. CEAP clinical classification of chronic venous insufficiency (adapted from $[38,39])$.

\begin{tabular}{|c|c|c|}
\hline \multicolumn{2}{|c|}{ Klassifikation } & \multirow{2}{*}{$\frac{\text { Definition }}{(a=\text { asymptomatisch, } s=\text { symptomatisch) }}$} \\
\hline C & Clinic & \\
\hline $\mathrm{CO}$ & & $\begin{array}{l}\text { Keine sichtbaren oder palpablen Zeichen einer } \\
\text { venösen Erkrankung }\end{array}$ \\
\hline $\mathrm{C} 1$ & & $\begin{array}{l}\text { Teleangiektasien, retikuläre Venen, Corona } \\
\text { phlebectatica }\end{array}$ \\
\hline $\mathrm{C} 2$ & & Varikose \\
\hline $\mathrm{C} 3$ & & Ödem ohne Hautveränderung \\
\hline \multirow[t]{3}{*}{$\mathrm{C} 4$} & & Hautveränderungen \\
\hline & C4a & Pigmentation, Stauungsekzem oder beide \\
\hline & C4b & Dermoliposklerose, Atrophie blanche oder beide \\
\hline $\mathrm{C} 5$ & & Hautveränderungen mit geheiltem Ulkus \\
\hline $\mathrm{C} 6$ & & Hautveränderungen mit floridem Ulkus \\
\hline $\mathbf{E}$ & Etiology & $\begin{array}{l}\text { Kongenital (seit Geburt vorhanden), primär } \\
\text { (unklare Ätiologie), sekundär (postthrombotisch, } \\
\text { traumatisch) }\end{array}$ \\
\hline A & Anatomy & $\begin{array}{l}\text { Oberflächlich: V. saphena magna, V. saphena parva } \\
\text { Tiefe Venen: V. cava - Unterschenkelvenen } \\
\text { Perforansvenen }\end{array}$ \\
\hline $\mathbf{P}$ & Pathophysiology & Reflux - Obstruktion \\
\hline
\end{tabular}

nösen Wiederauffüllzeit vor und nach Abbinden von Varizen bzw. Kompression insuffizienter Perforansvenen kann der postinterventionelle Zustand simuliert werden [41]. Eine häufig angewandte Methode ist die Air-Plethysmographie, die auf dem Verschieben von Luftvolumen in einer um die Wade angelegten Manschette während Manövern zum Füllen bzw. Entleeren des venösen Systems basiert. Sie erlaubt, Reflux bzw. Obstruktion der Beinvenen sowie die Dysfunktion der Muskelpumpe zu studieren [42].

Phlebodynamometrie. Der Druck in einer Fußrückenvene wird blutig zuerst am ruhig stehenden Patienten und dann während eines standardisierten Be-

Tabelle 4. Differentialdiagnostik von arteriellem, venösem und neuropathischem Ulkus (modizifiert nach [40]).

Table 4. Differential diagnosis of arterial, venous and neuropathic ulcer (adapted from [40]).

\begin{tabular}{llll}
\hline Charakteristika & Arterielles Ulkus & Venöses Ulkus & Neuropathisches Ulkus \\
\hline Lokalisation & Zehen oder Fuß & Malleolär oder metatarsal & Häufig an Fußsohle \\
Erscheinungsbild & Irregulärer Rand, & Irregulärer Rand, rosarot, & Ausgestanzt, rot, \\
& blass oder zyanotisch & tiefliegend und infiziert & Überwärmt, trocken \\
Temperatur & Kühl & Überwärmt & Fehlend \\
Schmerz & Zum Teil massiv & Leichtgradig & Palpabel \\
Fußpulse & Fehlend & Palpabel & Dilatiert \\
Venen & Kollabiert & Dilatiert, varikös & Fehlend \\
Sensibilität & Wechselhaft & Vorhanden & Vorhanden \\
Kallus & Fehlend oder selten & Fehlend & Oft vorhanden \\
Knochendeformität & Fehlend & Fehlend & \\
\hline
\end{tabular}


wegungsprogramms gemessen [43]. Die Phlebodynamometrie ist die bestreproduzierbare und exakteste Methode aller venösen Funktionsmessungen. Sie ist allerdings invasiv, deshalb nicht beliebig wiederholbar und bleibt nur für spezielle Fragestellungen reserviert.

\section{Apparative Diagnostik - morphologische Tests}

Phlebographie. Die aszendierende Phlebographie wird als Goldstandard zur Beurteilung der Venendurchgängigkeit angesehen. Bei der deszendierenden Phlebographie wird Reflux durch proximale Injektion von Kontrastmittel unter Valsalva-Manöver studiert [44]. Die Untersuchung liefert objektive anatomische und hämodynamische Informationen über das Venensystem. Wie bereits im Abschnitt Varikose erwähnt, überwiegen die Nachteile wie Invasivität, Strahlenexposition, mögliche Kontrastmittelunverträglichkeit oder kontrastmittelinduzierte Niereninsuffizienz und nicht zuletzt hohe Kosten, so dass die Phlebographie weitgehend durch nichtinvasive Verfahren abgelöst wurde. Trotzdem hat die Methode bei gewissen Situationen noch einen festen Platz, insbesonders vor einer aktiven chirurgischen oder interventionellen therapeutischen Maßnahme [45].

Duplexsonographie. Wie in der Diagnostik der TVT wird die Duplexsonographie heutzutage routinemäßig zur Bestätigung der chronischen venösen Insuffizienz und zur Abschätzung deren Ursache bzw. Schweregrades eingesetzt (Abbildung 5b). Bereits in den 80er Jahren stellten Szendro et al. fest, dass die Methode bei der Beurteilung von Klappeninsuffizienzen im Vergleich zur blutigen Venendruckmessung als Goldstandard mit einer Sensitivität von $84 \%$ und einer Spezifität von $88 \%$ recht gut abschneidet [46]. Bezüglich der Feststellung des Schweregrades der chronischen venösen Insuffizienz ist sie der deszendierenden Phlebographie sogar überlegen [47].

\section{Apparative Diagnostik - weitere diagnostische Tests}

Ankle-Brachial-Index (ABI). Patienten mit chronischer venöser Insuffizienz und Ulcus cruris können konkomitierend eine periphere arterielle Verschlusskrankheit aufweisen. Ein gemischt arteriovenöses Ulkus kommt in 20-30\% der Patienten mit Ulcus cruris vor, also wesentlich häufiger als allgemein angenommen [48, 49]. In der Diagnostik der peripheren arteriellen Verschlusskrankheit hat sich die Messung arterieller Knöcheldrücke mittels eines einfachen Taschen-Dopplers bewährt. Bei einem ABI (höchster am Knöchel gemessener Druckwert dividiert durch den höchsten Oberarmdruck) $<0,9$ liegt eine periphere arterielle Verschlusskrankheit vor [50].

Bei Patienten mit Ulcus cruris und gleichzeitig fehlenden Fußpulsen sollte diese einfache Messung unbedingt durchgeführt werden, um eine gemischt arteriovenöse Genese nicht $\mathrm{zu}$ verpassen, da diese Patientengruppe therapeutisch potentiell von einer arteriellen Revaskularisation profitieren würde.

Hautbiopsie. Bei Vorliegen einer chronischen venösen Insuffizienz wurden komplexe histologische Veränderungen in Kapillaren, lymphatischen Bahnen und in Hautgewebe gefunden [51, 52]. In klinischem Alltag empfiehlt sich in gewissen Fällen eine Hautbiopsie, primär nicht zur Bestätigung der venösen Diagnose, sondern eher zum Ausschluss eines Malignoms.

Interessenkonflikt: Es besteht kein Interessenkonflikt. Die Autoren versichern, dass sie keine finanziellen oder persönlichen Beziehungen zu Dritten haben, deren Interessen das Manuskript positiv oder negativ beeinflusst haben könnten.

\section{Literatur}

1. Evans CJ, Fowkes FGR, Ruckley CV, et al. Prevalence of varicosis veins and chronic venous insufficiency in men and women in the general population: Edinburgh Vein Study. J Epidemiol Community Health 1999;53:149-53.

2. Partsch H. Update on chronic venous insufficiency: from epidemiology to management. Angiology 2001;52:1-25.

3. Widmer L. Venenkrankheiten. Häufigkeiten und sozialmedizinische Bedeutung. Basler Studie III. Bern: Huber, 1982.

4. McMullin GM, Coleridge Smith PD. An evaluation of Doppler ultrasound an photoplethysmography in the investigation of venous insufficiency. Aust N Z J Surg 1992;62:270-5.

5. Jeanneret C, Lack S, Labs K, et al. Intraindividuelle Variabilität des mit standardisiertem Valsalva-Manöver erzeugten venösen Reflux bei Venengesunden. Vasa 1999;28:223-38.

6. Van Bemmelen P, Bedford G, Beach K, et al. Ouantitative segmental evaluation of venous valvular reflux with duplex ultrasound scanning. J Vasc Surg 1989;10:425-31.

7. Labropoulos N, Tiongson J, Pryor L, et al. Definition of venous reflux in lower-extremity veins. J Vasc Surg 2003;38:793-8.

8. Verlato F, Zucchetta P, Prandoni P, et al. An unexpectedly high rate of pulmonary embolism in patients with superficial thrombophlebitis of the thigh. J Vasc Surg 1999;30:1113-5.

9. McColl M, Ramsay J, Tait R, et al. Superficial vein thrombosis. Incidence in association with pregnancy and prevalence of thrombophilic defects. Thromb Haemost 1998;79:741-2.

10. Elting LS, Escalante CP, Cooksley $\mathrm{C}$, et al. Outcomes and cost of deep venous thrombosis among patients with cancer. Arch Intern Med 2004;164:1653-61.

11. Khorana AA, Francis CW, Culakova E, et al. Thromboembolism in hospitalized neutropenic cancer patients. J Clin Oncol 2006;24:484-90.

12. Stein PD, Beemath A, Meyers FA, et al. Pulmonary embolism as a cause of death in patients who died with cancer. Am J Med 2006;119:163-5.

13. Cushman M, Tsai AW, White RH, et al. Deep vein thrombosis and pulmonary embolism in two cohorts: the longitudinal 
investigation of thromboembolism etiology. Am J Med 2004;117:19-25.

14. Partsch H, Oburger K, Mostbeck A, et al. Frequency of pulmonary embolism in ambulant patients with pelvic vein thrombosis: a prospective study. J Vasc Surg 1992;16:715-22.

15. Kahn SR, Ginsberg JS. The post-thrombotic syndrom: current knowledge, controversies, and directions for future research. Blood Rev 2002;16:155-65.

16. Birdwell BG, Raskob GE, Whitsett TL, et al. The clinical validity of normal compression ultrasonography in outpatients suspected of having deep venous thrombosis. Ann Intern Med 1998;128:1-7.

17. Hull RD, Hirsh J, Sackett DL, et al. Clinical validity of a negative venogram in patients with clinically suspected venous thrombosis. Circulation 1981;64:622-5.

18. Constans J, Boutinet C, Salmi LR, et al. Comparison of four clinical prediction scores for the diagnosis of lower limb deep venous thrombosis in outpatients. Am J Med 2003; 115:436-40.

19. Wells PS, Anderson DR, Bormanis J, et al. Value of assessment of pretest probability of deep vein thrombosis in clinical management. Lancet 1997;350:1795-8.

20. Bounameaux $\mathrm{H}$, de Moerloose P, Perrier A, et al. Plasma measurement of D-dimer as diagnostic aid in suspected venous thromboembolism: an overview. Thromb Haemost 1994; 71:1-6.

21. Keeling DM, Mackie IJ, Moody A, et al. The diagnosis of deep vein thrombosis in symptomatic outpatients and the potential for clinical assessment and D-dimer assays to reduce the need for diagnostic imaging. $\mathrm{Br} J$ Haemato 2004;124:15-25

22. Wells PS, Anderson DR, Rodger $M$, et al. Evaluation of D-dimer in the diagnosis of suspected deep-vein thrombosis. N Engl J Med 2003;349:1227-35.

23. Didier D, Racle A, Etievent JP, et al. Tumor thrombus of the inferior vena cava secondary to malignant abdominal neoplasms: US and CT evaluation. Radiology 1987;162:83-9.

24. Mood AR, Pollock JG, O'Connor AR, et al. Lower-limb deep venous thrombosis: direct MR imaging of the thrombus. Radiology 1998;209:349-55.

25. Lensing AW, Buller HR, Prandoni P, et al. Contrast venography, the gold standard for the diagnosis of deep-vein thrombosis: improvement in observer agreement. Thromb Haemost 1992;67:8-12.

26. Couson F, Bounameaux C, Didier D, et al. Influence of variability of interpretation of contrast venography for screening of postoperative deep venous thrombosis on the results of a thromboprophylactic study. Thromb Haemost 1993; 70:573-5.

27. Douglas MG, Sumner DS. Duplex scanning for deep vein thrombosis: has it replaced both phlebography and noninvasive testing ? Semin Vasc Surg 1996;9:3-12.

28. Donnelly R, Hinwood D, London NJ. ABC of arterial and venous disease. Non-invasive methods of arterial and venous assessment. BMJ 2000;320:698-701.

29. Birdwell BG, Raskob GE, Whitsett TL, et al. Predictive value of compression ultrasonography for deep vein thrombosis in symptomatic outpatients: clinical implications of the site of vein noncompressibility. Arch Intern Med 2000;160:309-13.

30. Elias A, Colombier D, Victor G, et al. Diagnostic performance of complete lower limb venous ultrasound in patients with clinically suspected acute pulmonary embolism. Thromb Haemost 2004;91:187-95.

31. Schellong SM, Schwarz T, Halbritter K, et al. Complete compression ultrasonography of the leg veins as a single test for the diagnosis of deep vein thrombosis. Thromb Haemost 2003;89:228-34.
32. Labropoulos N. Hemodynamic changes according to the CEAP classification. Phlebolymphology 2003;40:130-6.

33. Kistner RL, Eklof B, Masuda EM. Diagnosis of chronic venous disease of the lower extremities: the "CEAP" classification. Mayo Clin Proc 1996;71:338-45.

34. Abenhaim L, Kurz X, Norgren L, et al. The management of chronic venous disorders of the leg: an evidence-based report of an international task force. Phlebology 1999;14: Suppl 1:1-126.

35. Kurz X, Kahn SR, Abenhaim L, et al Chronic venous disorders of the leg: epidemiology, outcomes, diagnosis and management: summary of an evidence-based report of the VEINES Task Force. Int Angiol 1999;18:83-102.

36. Scott TE, LaMorte WW, Gorin DR, et al. Risk factors for chronic venous insufficiency: a dual case-control study. J Vasc Surg, 1995;22:622-8.

37. Widmer LK, Stählin HB, Nissen C, et al. Venen-, ArterienKrankheiten, koronare Herzkrankheit bei Berufstätigen, prospektiv-epidemiologische Untersuchung. Baseler Studie I-III 1959-1978. Bern-Stuttgart-Wien: Huber 1978.

38. Porter JM, Moneta GL. Reporting standards in venous disease: an update. International Consensus Committee on Chronic Venous Disease. J Vasc Surg 1995;21:635-45.

39. Eklof B, Rutherford RB, Bergan JJ, et al. Revision of the CEAP classification for chronic venous disorders: consensus statement. J Vasc Surg 2004;40:1248-52.

40. Dormandy JA, Rutherford RB. Management of periphera arterial disease (PAD). TASC Working Group. TransAtlantic Inter-Society Concensus (TASC). J Vasc Surg 2000;31:Suppl 1:1-296.

41. Partsch H. „Besserbare“ und "nicht besserbare“ chronisch venöse Insuffizienz. Vasa 1980;9:165-7.

42. Criado E, Farber MA, Marston WA, et al. The role of air plethysmography in the diagnosis of chronic venous insufficiency. J Vasc Surg 1998;27:660-70.

43. Nachbur B. Die periphere Venendruckmessung. Zentralb Phlebol 1971;10:224-30.

44. Herman RJ, Neiman HL, Yao JST, et al. Descending venography: a method of evaluating lower extremity venous valvular function. Radiology 1980;137:63-9.

45. Savolainen $\mathrm{H}$, Toivio I, Mokka R. Recurrent varicose veins: is there a role for varicography? Ann Chir Gynecol 1988;77: 70-3.

46. Szendro G, Nicolaides AN, Zukowski AJ, et al. Duplex scanning in the assessment of deep venous incompetence. J Vasc Surg 1986;4:23-42.

47. Neglen P, Raju S. A comparison between descending phlebography and duplex Doppler investigation in the evaluation of reflux in chronic venous insufficiency: a challenge to phlebography as the "gold standard". J Vasc Surg 1992;16: 687-93.

48. Callam MJ, Harper DR, Dale JJ, et al. Arterial disease in chronic leg ulceration: an underestimated hazard? Lothian and Forth Valley leg ulcer study. Br Med J 1987;294:929-31.

49. Baker SR, Stacey MC, Singh G, et al. Aetiology of chronic leg ulcers. Eur J Vasc Surg 1992;6:245-51.

50. Barnes RW. Noninvasive diagnostic assessment of peripheral vascular disease. Circulation 1991;83:Suppl I:I.

51. Leu HJ. Morphology of chronic venous insufficiency: light and electron microscopic examinations. Vasa 1991;20: 330-42.

52. Herrick SE, Sloan P, McGurk M, et al. Sequential changes in histologic pattern and extracellular matrix deposition during the healing of chronic venous ulcers. Am J Pathol 1992;141:1085-95

\section{Korrespondenz- anschrift}

Prof. Dr. Dai-Do Do

Klinik und Poliklinik für Angiologie

Departement Herz und Gefäße

Inselspital Universität Bern 3010 Bern

Schweiz

Telefon (+41/31)

632-3034, Fax -4793

E-Mail:dai-do.do@

insel.ch 